\title{
THE GEOLOGICAL HISTORY OF POLYPLOIDY IN WOODY ANGIOSPERMS
}

MASTERSON, Jane, Committee on Evolutionary Biology, University of Chicago, 5734 S. Ellis Ave., Chicago, IL 60637 U.S.A

Polyploidy, the condition where an organism has more than two complete sets of chromosomes, is a common and important evolutionary phenomenon in flowering plants (angiosperms).

Estimates of the percentage of angiosperms (approximately 250-300,000 living species) derived from polyploids range from $47-90 \%$. The historical development of polyploidy is currently very poorly understood and whether polyploidy is a major causal factor in angiosperm diversification is widely debated.

Many questions about the base chromosome number of angiosperms (important for establishing the extent of polyploidy) and the origin of polyploidy can be addressed using fossil plants. One of the physical effects of polyploidy is a general increase in cell volume; the size of guard cells (leaf epidermal cells that control the opening and closing of the gas exchange pores [stomata]) has been used successfully to determine the relative number of chromosomes present or ploidy level in living Carya (hickories), Betula (birch), Magnolia, and Pyrus (pear). At least two previous studies have used guard cells to estimate ploidy level in fossil taxa (Metasequoia, Sequoia, and Paleorubiaceophyllum).

The base haploid chromosome number in angiosperms generally has been assumed to be between $n=7-9$, but this is apparently based either on the dubious assumption that the most common condition is likely to be primitive (cf. egg laying in mammals) or the inference that because this number is found in many different clades it is likely to be basic in each of these groups. The Gnetales (the living gymnosperms most closely related to angiosperms) have a haploid basic number of 7, but Donoghue and Doyle (1989) find that it is almost equally parsimonious to assume the base number is $n=12-14$ because of uncertainties over the chromosome number of certain critical extinct outgroups. My guard cell measurements of three angiosperm families (Platanaceae, Magnoliaceae and Lauraceae) provide additional empirical support for the $n=7-9$ hypothesis and suggest that extinct diploid members existed in the midCretaceous and early Cenozoic floras. These results also falsify the hypothesis that polyploidization triggered the angiosperm radiation in the Cretaceous in these families. Other hypotheses regarding the historical timing of major increases in polyploidy are being tested. These include climatic deterioration and associated modification and mixing of plant distributions during the Eocene and Pleistocene. It may be that polyploids survive major disturbances at a higher rate than diploids because they often have broader ecological tolerances and wider geographic ranges. Thus the accumulation of polyploids in plant families may not occur because they are speciation prone as usually inferred but because they are extinction resistant. My preliminary results in Platanaceae support an increased level of polyploidy after major disturbances. 